\title{
Computer Network Security Analysis and Research Limei Cui
}

\author{
Qujing normal college, yunnan qujing 655001
}

\section{Keywords: Computer; Network; Security}

\begin{abstract}
In today's era is the era of information technology rapid development, with the constant progress of the society, the network technology is in constant development, now the network is essential in our daily life people less a thing. Using the network, at home the whole messy, never leave home can know the global access to rich information resources around the world. In the modern economic, military and all kinds of social activities are also particularly dependent on network technology. A computer is an integral part of People's Daily life, in daily office is now dependent on the computer. But computers and the Internet is a double-edged sword, in the benefits at the same time also caused the overflow of the hacker, the loss of personal information, let the computer network security issues are the great threat. But, we also made efforts to make our network security guarantee.

Computer network security is refers to the use of network management technology and control measures to ensure that, in a network environment data confidentiality, integrity and usability are protected. Computer network security includes physical security and logical security from two aspects, including physical security refers to the physical system equipment and related equipment protection, prevent loss; Logic security includes information confidentiality, integrity, and availability.
\end{abstract}

\section{Computer Network Security Threats}

A computer virus. A computer virus is a weaver in a computer program insert damage computer functions or data code, computer virus can affect the normal use of the computer, computer viruses can replicate. Computer virus spread fast, it just like a biological virus can reproduce, activate the regeneration or infect each other. Computer virus replication ability is unique, it spreads quickly, but when it is difficult to remove, so destructive. They tend to be attached to all kinds of the type of the file, the file replication and transmission between the spread.

In computer viruses typically have 1986 Pakistan brothers to write the "brain" of the virus, "small ball" and "stone" in 1987 virus, "stone 2" in 1989, in 1992 the "golden cicada" virus, 1995 years of a typical representative "virus making machine" "the VCL", "CIH virus" in 1988 and after 2000 "shockwave killer" "Trojan horse" "hackers" virus "worm" "cover letter" and so on the power of these viruses are very large.

The hacker's attack. With the rapid development of Internet hacking techniques, the threat of computer network is more and more serious, for hacking into your computer for your information is a very easy thing, the hacker attack means can be divided into non destructive attacks and destructive attacks, in either attack for the harm is very big. The hacker attack method respectively is: comparison of several commonly used backdoor, information bomb, denial of service, network monitoring, and password cracking. Maintaining network security's main goal is to ensure data confidentiality, integrity. In a computer network security environment, unauthorized data is can't literally change of want to get any data information personnel should be authorized legitimate users.

According to the 2008 rising in mainland China Internet security report shows, for the purpose of making profits hacker attacks has formed the industrial chain, became the new profiteering industry, in China a few years ago some of the important department had suffered the hacker's attack, some government departments, military industrial enterprises and other important units in a large network hacker attacks.

Internal threats. Owing to lack of Internet access unit for the understanding of the internal risk comprehensive security measures taken by the improper, increasing the internal network safety 
accident in recent years. Think of unintentional mistake is one of the important factors of network security threat; the network administrator role in this respect, a bit of a consideration of indiscretion security configuration inappropriate can cause larger security vulnerabilities. If there are some security vulnerabilities in the network, the user click when surfing on the net the malicious link can cause safety issues in the file.

Eavesdropping. Attackers through to the network data monitoring and access to sensitive information caused the leakage of information, the main performance of hacking is eavesdropping on the network information. More malicious attacker on this basis, using some other means of attack, caused more losses.

\section{Computer Network Security Countermeasures}

To Strengthen the Security of Network Technology. For the user identity verification, ensure the authenticity of the user's identity. Such as face recognition, public key encryption authentication, fingerprint identification, etc., to guarantee network security. To strengthen the confidentiality of information, to ensure that the confidential information is not leaked to not authorized person, permissions for different staff, without permission will not be able to access, this can prevent eavesdropping intercept and network. It is need to strengthen the integrity of the information for not authorized personnel is not able to modify or delete data or information, strictly control the access of information data, can only be allowed after approval in the party to modify, and delete. Network security should also have a system to use, can review. System usability refers to under the condition of can meet the safety requirements, the operation of the system should be easy to learn, easy to operate. Can review sex refers to the problems of the system can provide the basis of investigation and means.

To Strengthen the Network Access Control. Access control system is indispensable in a safe information system; its purpose is to refuse illegal user's access and specification for legitimate users of operation. The first layer of access control network access is net access control. It can allow users to access the time, to allow them in which a time period into the net, and makes some users will be able to login to the server and access to network resources. Access control can be according to the user's permission to give this user access, unlimited access to resources, prevent the users make every visitor's operation under the monitoring of the system, and then let the legitimate use of resources. Network access control step is when a particular user's access request, access control to the user's ID and identity to the security policy database query, and if found users for a particular resource access request will allow the user to visit, refused to conversely.

Strengthen the Network Access Control. Network access control is illegal operation have put forward a kind of network security protection. Have certain permissions users can access some directory specified file or other resource, some specified users can these directories, files and other resources for a certain operation. We access permissions can be divided into special user, generally refers to the system administrator, is the system administrator user generally classified according to the actual needs of users, users of audit is the audit of network security and resource usage.

To Improve the Security of Network Education and Management. The United States as the world's countries with high levels of information technology, it attaches great importance to the security of information, the information system of his father's to one of the most important part of national security strategy, and adopted a series of policies and measures, so want to improve the security of computer network in our country, then formulate corresponding laws and regulations as the basis, to strengthen the education and management of network security. Countries should attach great importance to the calculation of network security and to vigorously popularize family education, develop and implement a series of network security laws and regulations, strengthen the network security awareness education for citizens and for network operation and network security management personnel of education, improve its quality and management measures, aimed at to raise awareness of the broad masses of users of network security and lower the nation's computer network security threats. To strengthen education about network security at the same time also must strengthen the supervision of network security, for malicious destruction of computer network 
security for a severe punishment, for no damage to carry on the education, establishing strict network security management system, timely detection of maintaining the security of the computer network.

Improve Network User Operation Skills. Along with the advance of information, computer network security for network users more and more serious damage, so the network user's operation skills is imperative. For improving network user's operation skill, the first is to strengthen the users of virus detection and defense, it goes on to identify the user's permissions, automatic open network protection and firewall functions, automatic killing virus, to improve the user's Internet skills. On the other hand is to pay attention to prevent external malicious attacks, access and download the health files, before the download for detection and virus scan files necessary, conghua a variety of measures to reduce the computer network security problems.

\section{Conclusion}

With the continuous development of computer technology, network security protection technology has also inevitably will continue to progress and development, network security is an involved in technology, management and use various comprehensive subject, so we have to consider. In today's complex under the network environment, we should highly attaches great importance to the computer network problems, enhance social security consciousness education, education and popularization of computer network security related departments to reinforce demands for network security supervision, improve the level of computer network security technology, actively build a security of the computer network environment construction, improving the security situation now.

\section{References}

[1] Earning speech, sum up experience 2.8\% (54) Guo Hancheng - "opera art" - 2012

[2] On the visual management of equipment 1\% (18) MA - "China management information" 2014

[3] Cai Xiaolian Li ping, the computer network security threats and protection strategy of study"

[4] Xu Xiaolong; Xiong Jingyi; Cheng Chunling. Journal of the joint defense mechanism of malicious code based on [J]. Cloud computing architecture of Southeast University (NATURAL SCIENCE EDITION), 201102.

[5] Liu Yang; Tang Zhuo; Li Renfa; Zhang Zongli. User access needs role search algorithm [J]. Journal of communication based on cloud computing environment, in 201107.

[6] Yellow crane "shallow of computer network security"

[7] Sun Zhikuan present situation and countermeasure of computer network security research

[8] Analysis of network accounting information system security issues $0.8 \%$ (16) Liang Xiaoqing "China's chief accountant" - 2013

[9] Lin Guo; He Shan; Huang Hao; Wu Jiyi; Chen Wei. Access control security model of [J]. communication behavior based on Cloud Computing Journal, 201203

[10] Was Chen Ping. Internet plus and innovation [J]. Journal of Xichang College (NATURAL SCIENCE EDITION), 2015,04:59-62. 